\title{
Chess endgame knowledge advances
}

\author{
Article
}

Accepted Version

Haworth, G. M. (2010) Chess endgame knowledge advances. ICGA Journal, 33 (3). p. 149. ISSN 1389-6911 Available at https://centaur.reading.ac.uk/16303/

It is advisable to refer to the publisher's version if you intend to cite from the work. See Guidance on citing.

Publisher: The International Computer Games Association

All outputs in CentAUR are protected by Intellectual Property Rights law, including copyright law. Copyright and IPR is retained by the creators or other copyright holders. Terms and conditions for use of this material are defined in the End User Agreement.

\section{www.reading.ac.uk/centaur}

\section{CentAUR}

Central Archive at the University of Reading

Reading's research outputs online 


\title{
NOTE
}

\section{CHESS ENDGAME KNOWLEDGE ADVANCES}

\author{
G.M $M^{\mathrm{C} C . ~ H a w o r t h}$ \\ Reading, UK
}

There are two major publishing events to report. First, the doyen of the Chess Study community, Harold van der Heijden (2010) has released edition 4 of his Study Database, an awesome collection of 76,132 studies with the best known ground truth about their correctness. Haworth and Bleicher used CQL, PGN2FEN and the Nalimov EGTs to check all the sub-7-man mainline positions that were to be included. They detected transcription errors, misstipulations, and errors in some 3,000 studies, about half of which were known. Van der Heijden et al. (2010) highlighted some of the more interesting 'escapes' that were revealed for Black.

Secondly, the matchless John Nunn $(2009,2010)$ has published his second trilogy on the endgame, dividing it not by endgame force but into zones. These are discussed within three themes: core knowledge, Rook endgames, and non-Rook endgames. John's writing is as straightforward and accessible as ever even for the casual enthusiast. His exemplar positions are carefully chosen to illustrate valuable concepts and principles of play: the analysis is highly assured, being supported by powerful chess engines and the full set of Nalimov EGTs.

Following Bleicher and Haworth (2010), Bleicher is identifying all sub-7-man Type B zugzwangs, i.e., positions in which theoretical value is not at stake but where the win is quicker if the side-to-move 'passes' with a null move. His results for depth-difference greater than 2 are available (Beasley and Bleicher, 2010) in Beasley's nomenclature and called squeezes by him. The task explodes in size when the depth-gain from 'passing' is merely positive.

In a similar but much larger exercise than that on van der Heijden's Study Database, Haworth and Bleicher have analysed sub-7-man play in the mainline of all games in Chessbase's BIG CHESSBASE 2010. So far, we have been able to identify all sub-7-man zugzwangs of Types A and B (as for studies), all games where the last position does not have the same theoretical value as the designated game-result ${ }^{2}$, the overall statistics of concessions of theoretical value, and the extreme examples of value-trading and zugzwangs.

The tasks above would have been greatly facilitated by the existence of EGTs for positions with castling rights. Although the move-count is not zeroed as it might be after an irreversible reduction in castling rights, positions with such rights may be thought of as in separate endgames and the computation of their EGTs is an additive and relatively small scale task. All positions in the endgame $K R R K R R \_K Q k q^{3}$ are, by the way, unreachable!

Haworth (2010) is making available an increasing range of sub-6-man EGTs for the DTC, DTZ and DTZ50 metrics. A related challenge is being addressed, that of creating an EGT-generation algorithm which can be transformed into efficient code across the anticipated range of distributed-architecture machines. Use across various metrics, depth in plies, and inclusion of castling rights and some variants of chess are on the agenda.

\section{References}

Beasley, J. and Bleicher, E. (2010). http://www.k4it.de/egtb/zugzwang.php Type A and B zugzwangs.

Bleicher, E. and Haworth, G.M ${ }^{\mathrm{c} C}$. (2010). 6-man chess and zugzwangs. Proceedings of Advances in Computer Games, 12 (2009). Lecture Notes in Computer Science, 6048, pp. 123-135. ISSN 0302-9743

Haworth, G.MC. (2010). http://extra.sse.reading.ac.uk/people/G.Haworth/ DTC, DTZ and DTZ50 EGTs.

Nunn, J. (2009). Understanding Chess Endgames. Gambit. ISBN 1-9064-5411-6.

Nunn, J. (2010). Nunn's Chess Endings, v1 \& v2. Gambit, ISBNs 1-9064-5421-3 and 1-9064-5423-X.

van der Heijden, H. (2010). http://www.hhdbiv.nl/ Chess Study database, edition 4.

van der Heijden, H., Bleicher, E. and Haworth, G.MC. (2010). Endgame Table Analysis of Chess Studies I-II, EG Vol. 16, No. 180, pp. 114-9 and No. 181, pp. 163-9.

\footnotetext{
${ }^{1}$ The University of Reading, Berkshire, UK, RG6 6AH. email: guy.haworth@bnc.oxon.org.

${ }^{2}$ The combination of DGT boards, software and the 'DGT' protocol of centring Kings may be responsible for many of these.

${ }^{3}$ KRRKRR_KQkq is defined to be the endgame \{position | position in KRRKRR and KQkq castling rights in place\}.
} 